\title{
Synthesis and analysis of coupler curves with combined planar cam follower mechanisms
}

\author{
U.S. Chavan ${ }^{1 *}$, S.V. Joshi ${ }^{2}$ \\ ${ }^{1}$ Department of Mechanical Engineering, Vishwakarma Institute of Technology, Pune, Maharashtra, INDIA \\ ${ }^{2}$ Department of Mechanical Engineering, Vishwakarma Institute of Technology, Pune, Maharashtra, INDIA \\ "Corresponding Author: e-mail:umeshschavan@rediffmail.com , umesh.chavan@vit.edu +91-020-2420194, Fax.+91-020-24280926
}

\begin{abstract}
Cam and follower mechanisms are widely used to convert a rotary input motion into a controlled reciprocating or oscillating motion as output in machines or robots. As this mechanism has an ability to provide unlimited variety of output motions. Many works are done on the synthesis of coupler curves or path generation using four bar mechanism. The present work investigates the alternative way of dealing with the path generation problems of industrial application by using cam mechanisms. Proposed a new mechanism which comprises four bar and cam follower mechanism, these mechanisms are combined together to obtain a single-input combined cam mechanism (CCM). Dimensional synthesis has been carried out by guiding the coupler point along predefined path (ellipse or straight line) of profile cutting machine. One of the objectives was to compare the path of coupler in four bar and CCM. Further, kinematic simulation of both mechanisms was performed to estimate the error and validate the proposed methodology. Results show that coupler point follows same path as ellipse or straight line profile in both mechanisms. Proposed CCM successfully applied to the complex path generation problems of profile cutting machine like square, circle and figure eight profiles. A method of kinematic analysis of proposed mechanism was also discussed. Finally, dynamic stability and separation of cam follower contact was simulated by using Matlab Simulink tool. Results show that proposed CCM was found stable from kinematic and dynamic point of view.
\end{abstract}

Keywords: Cam and follower; Coupler; Dynamic; Fourbar Kinematic; Mechanism; Path generation

\section{Introduction}

In several applications, design of mechanical systems involves the synthesis of mechanisms in order to meet a set of kinematic requirements. Several methods of synthesis have been proposed by various authors for selecting and scaling mechanical devices. Graphical methods proposed by Hartenberg and Denavit (1964) and Sandor and Erdman (1984) are useful for limited number of precision points in synthesis. Freudenstein (1954) and Erdman (1981 and 1995) developed analytical approach for synthesis of planar mechanisms. Sandor and Erdman (1991) and Angeles and Cajin (1988) presented a computer aided numerical methods for synthesis of planar four bar and cam mechanisms. In all above methods, objective was to guide a rigid body through a series of specified positions (rigid body guidance) or to obtain a specific input and output relationship (function generation), or to force a point on a linkage to move along a prescribed trajectory (path generation), but these methods restrict the number of precision points or coordinates. Increase in computer power has permitted the recent development of routines that apply any number of precision points for path generation. Genetic algorithm (GA) is one of the recent techniques of kinematic synthesis. Genetic algorithms were first introduced by Holland (1975). Goldberg (1989) revealed that GA's can successfully apply to different engineering optimization problems. Laribi et al. (2004) cited a method based on genetic algorithms and fuzzy logics for the synthesis of four bar mechanisms and combined cam linkages. First step is based on crank and rocker mobility criterion then, mechanism is considered with as many as degrees of freedom required by the generation task. A combined cam-linkage mechanism synthesized for precise motion, function and path generation. Mundo et al. (2006) proposed a genetic algorithm based method for the optimal synthesis of planar mechanisms. Used combined cam linkage for path generation task, and also some applications of this methodology are presented. Gabrera et al. (2000) applied genetic algorithms to synthesize the four bar 
mechanisms for path generation problem. Objective function was to minimize distance between coordinates of prescribed and designed. Lampein (2003) described the genetic algorithms for optimize the cam mechanisms, and then this analogy is applied to synthesize the automotive valve trains. The work presented by Fang (1994) deals with solution methods of optimal synthesis of planar mechanisms. A searching procedure is based on evolutionary techniques that are genetic algorithms. Problems of four-bar mechanisms are used to test the method. Singh and Kohli (1981) cited the existence of cam-linkage mechanisms for motion and path generation; used the complex loop closure method and the envelope theory to define a general approach for the synthesis of combined cam-linkage systems for exact path or motion generation. Sadler and Yang (1990) proposed a method of dynamic optimization of cam-linkage mechanisms. Recently Ullah and Kota (1991), Saggere and Kota (2001) and Smith and Ye (2005) used an analytical approach to the design of mechanical systems, where planar linkages are combined with a cam for driving an oscillating roller follower. Saxena (2005) and Kunjur and Krishnamurthy (1997) presented genetic algorithm approach to synthesize the mechanisms, also shown some results obtained by evolutionary techniques. These methods define a starting population that is improved by approximations of design variables. Gen and Cheng (2000) applied genetic algorithms to different engineering optimization problems. Also usefulness of this approach and advantages over the conventional optimization methods are mentioned. Different methods of kinematic analysis of planar mechanisms are described by Ghosh and Malik (2002) and Shigley and Uicker (1980). This analysis is very essential to study the motion of various links of a mechanism. Norton (2002) and Chen (1982) studied synthesis, analysis and dynamics of cam mechanisms. Cam mechanism can generate complex coordinated movements, relatively compact and easy to design. Recently Hafez and Su (2010) discussed the Synthesis of a slider-crank fourbar linkage is presented whose coupler point traces a set of predefined task points. There are at most 558 slider-crank four-bars in cognate pairs passing through any eight specified task points. The problem is formulated for up to eight precision points in polynomial equations. Umesh et al. (2010) presented Synthesis of coupler curves with combined planar cam follower mechanisms by genetic algorithm. Synthesis and path generation by single input combined cam mechanism using genetic algorithm was presented. Also this method applied to the complex path generation problem.

The objective of this work is to propose a novel CCM and a method of precise path generation with infinite number of precision points. Combine the conventional four bar and cam-follower mechanisms to obtain a CCM and dimensional synthesis. Then estimate the error in path generation by CCM and four bar. Finally carry out the kinematic and dynamic simulation to verify the stability of CCM by considering the various cases of cutting profiles or shapes. The paper is organized as follows; it presents a detailed literature survey in this field in the recent period. From the literature survey the main objectives were formed. Profile of coupler curves were defined, a four bar mechanism for dimension synthesized and combined with cam follower mechanism to get CCM. Subsequently to validate CCM different curves were generated. Then kinematic and dynamic competence of the developed CCM has been discussed.

\section{Formulation of a coupler curves}

Four bar is a kinematic equivalent mechanism of profile cutting machine. As coupler point defines the path of cutter, it is essential to guide this point along a desired cutting profile. Here, objective was to generate different coupler curves or cutting profiles such as an ellipse, square, circle and 8 shapes. Precision points (coordinates or target points) of elliptical shape is defined in Table I

Table I. Precision points

\begin{tabular}{|c|}
\hline Precision points $x_{\mathbf{z}}: 28,29,33,38,44,50,57,63,68,74$, \\
$77,79,79,78,75,70,65,59,53,45,41,35,30,27,26,25,28$ \\
Precision points $y_{2}: 11,12,15,18,24,32,34,41,47,53,58$, \\
$62,64.5,65.5,65,63,60,55,49,42,36,29,24,19,15,13,11$ \\
\hline
\end{tabular}

Dimensional synthesis of four bar mechanism has been carried by guiding coupler point along this pre defined shape. Synthesis was done without violating Grashoff's condition (Denavit, 1964, Sandor and Erdman, 1984 and 1991, Freudenstein, 1954 and Erdman, 1995 and 1981). As per Grashoff's criterion of crank and rocker (fourbar), summation of shortest and longest link length always less than the summation of remaining two link length. Constraint in dimensional synthesis was Grashoff's condition. Dimensions of four bar mechanism shown in Fig.1, were obtained by genetic algorithm (Goldberg, 1989, Laribi et al., 2004, Mundo et al., 2006, Gabrera et al., 2000, Lampein, 2003, Fang, 1994, Saxena 2005, Kunjur and Krishnamurthy, 1997).

\section{Synthesis of combined cam mechanism - CCM}

Aim was to guide a coupler point along an elliptical path, defined by a set of 27 precision points; this specific path generation problem was the interest of industrial profile cutting machine. Two independent parameters $\left(x_{t}\right.$ and $\left.y_{t}\right)$ are set for each configuration of the mechanism. It means control two independent parameters of each precision configuration. Therefore, the generation task requires a two DOF (degrees of freedom) mechanism (Laribi et al., 2004, Mundo et al., 2006, Gabrera et al. 2000). 
To get two DOF mechanisms, four bar (Fig.1) has been used by adding slider at the pair ' $\mathrm{E}$ '. A two DOF mechanism is shown in a Fig.2 where the slider displacement $S$ is added to the crank rotation $\theta_{2}$ as a second independent parameter, it allows the coupler point ' $\mathrm{T}$ ' precisely follow the predefined coupler curves shapes.

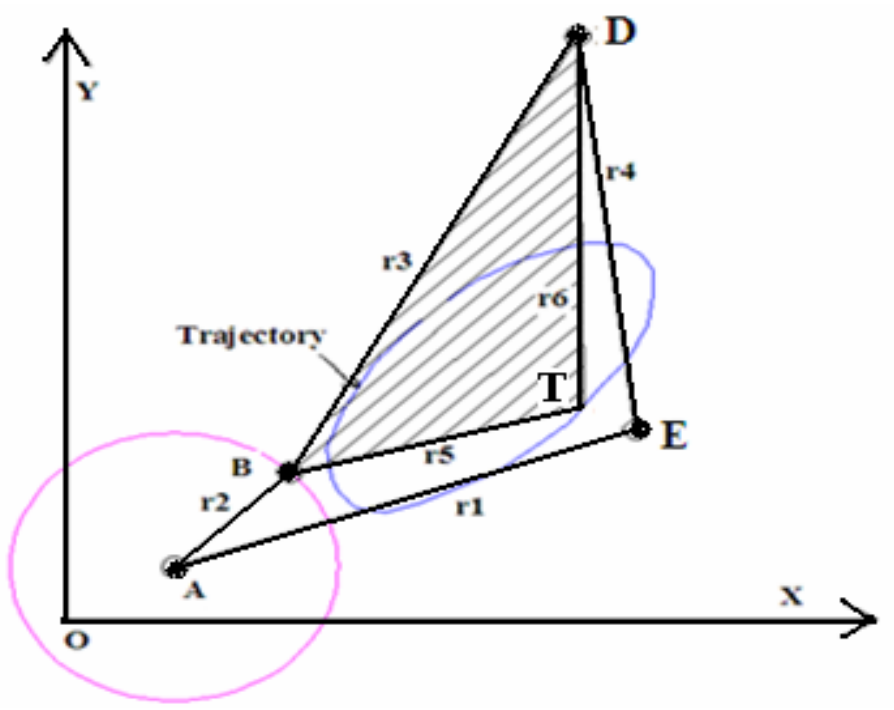

Figure 1. Crank and rocker four bar mechanism

Loop closure equation of fourbar mechanism

$r_{1}+r_{2}+r_{3}+r_{4}=0$

$s+r_{2}+r_{3}+r_{4}=0$

Loop closure equation of fourbar-slider

$s e^{i \theta_{1}}+r_{2} e^{i \theta_{2}}+r_{3} e^{i \theta_{3}}+r_{4} e^{i \theta_{4}}=0$

$r_{2} \cos \theta_{2}+r_{3} \cos \theta_{3}+r_{4} \cos \theta_{4}=s$

$r_{2} \sin \theta_{2}+r_{3} \sin \theta_{3}+r_{4} \sin \theta_{4}=0$

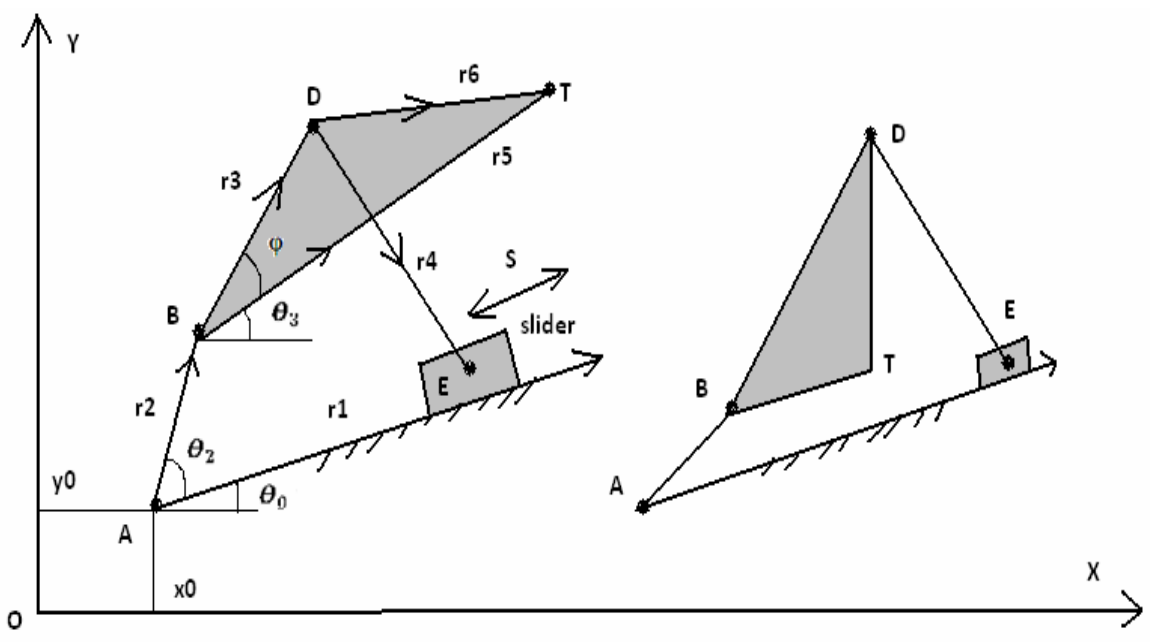

Figure 2. Four bar-slider mechanism - 2 DOF

From a mechanical point of view, the required correlation between $S$ and $\theta_{2}$ can be performed by synthesizing a cam follower mechanism, in which the rotational input is $\theta_{2}$ itself and translational output represented by $s$. Cam profile was synthesized on the basis of slider displacement (Singh and Kohli, 1981, Sadler and Yang, 1994, Ullah and Kota, 1997, Ye and Smith, 2005). Solve 
equations (1-5) for the variables $\theta_{2}, \theta_{3}, \theta_{4}, s$ using Matlab programming. Linkage dimensions and precision positions are same as of four bar mechanism. Input $\theta_{2}$ and slider displacement $s$ plotted in a Fig.3. Cam profile can be obtained by inverse kinematic from slider displacement. Cam profile for elliptical shape is shown in Fig.4. For industrial application one DOF mechanisms are more useful and economical. Therefore, to reduce the DOF of the mechanism (Fig.2) to one, crank was replaced by cam and slider as a follower. The independent parameters made dependent on cam follower motion. The consequent proposed combined cam mechanism (CCM) is represented in Fig.5. Pair 'B', where the cam and coupler are connected, 'A' being their center of rotation and ' $\mathrm{E}$ ' was a sliding pair.

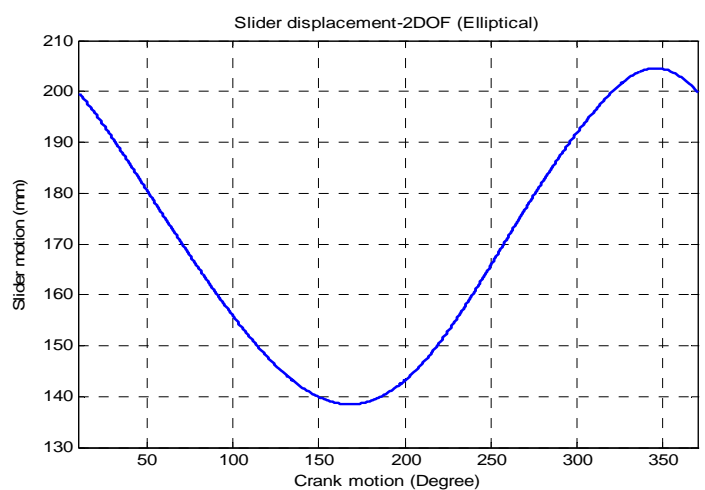

Figure 3. Slider displacement positions (s)

Synthesized four-bar mechanism satisfies Grashoff's criteria and offers a good performance. Same configuration was combined with cam and follower for generating the coupler curves. Dimensions obtained by Matlab programming, maximum displacement of follower $S_{\max }=203.2812 \mathrm{~mm}$, lowest position of follower $S_{\min }=139.3103 \mathrm{~mm}$ Stroke $h=S_{\max }-S_{\min }=63.9707 \mathrm{~mm}$. Basecircle radius of cam $S_{\min }$ and a maximum pressure angle of $28^{0}$.

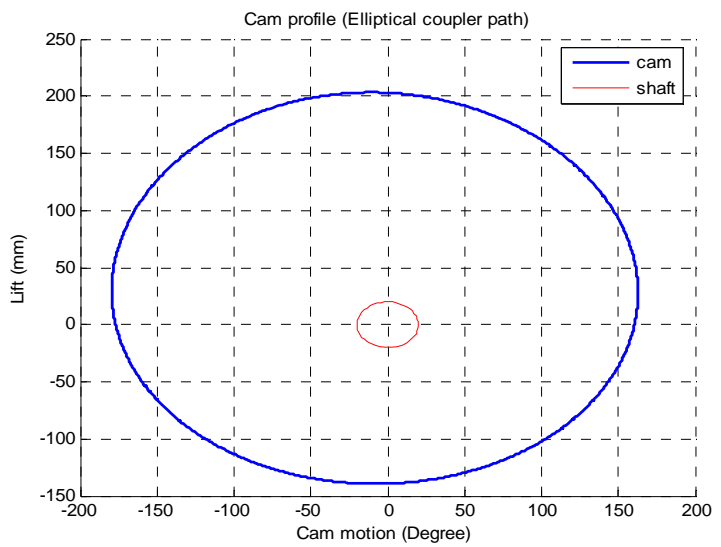

Figure 4. Cam profile

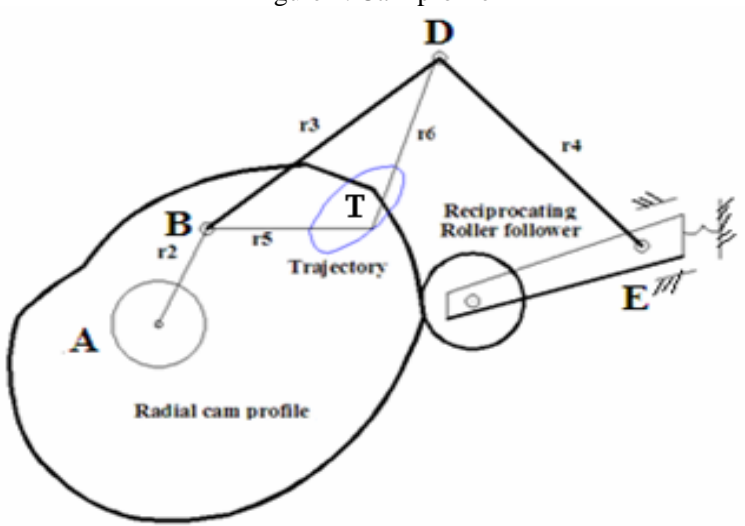

Figure 5. Proposed combined cam mechanism (CCM) 


\section{Results and discussion on generation of coupler curves}

Ellipse: Generated by both mechanisms as shown in a Fig.6. Kinematic simulation result shows that coupler point passes through the all precision points that have been specified by the elliptical path. Total structural error with respect to precision points in proposed CCM in $x_{t}\left(c_{x}\right) 2.8231$ and $y_{t}\left(c_{y}\right)-2.6$ and global error was 0.0497736 . This error value is within acceptable limit (Laribi et al., 2004, Mundo et al., 2006). Therefore proposed CCM found suitable for path generation applications.

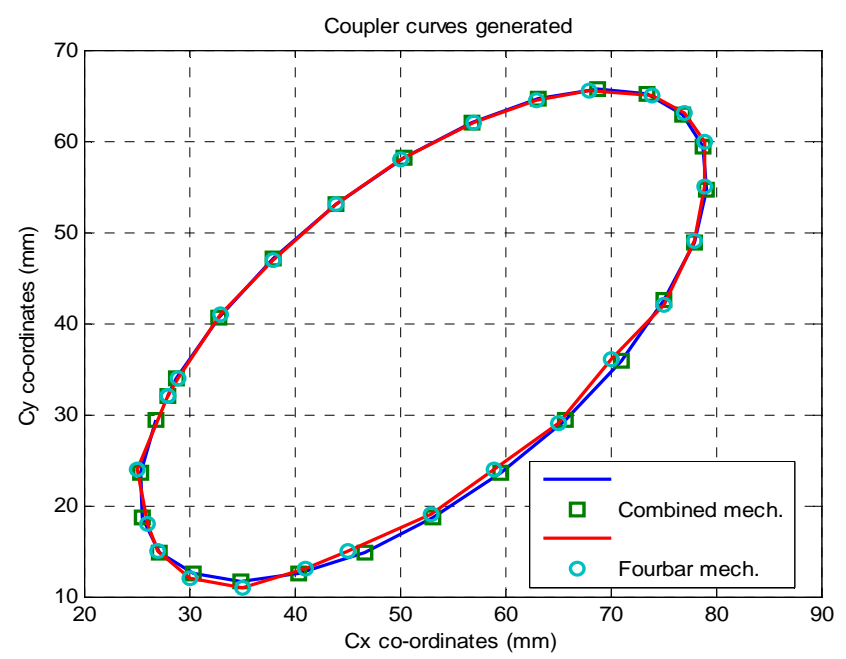

Figure 6. Path of coupler point

Complex path generation: To validate the application of this proposed methodology, some cases are simulated.

Straight line: Fig.7 shows straight line generated by four bar and CCM. It is observed that total error occurred in $x_{t}$ and $y_{t}$ coordinates were 0.00089 and 0.015 respectively, which are within acceptable limit.

Square and 8 or double circle profile: For square and double circle coupler curves, only multi DOF mechanisms are suitable (Laribi et al., 2004). But in proposed method one DOF CCM generated these curves. This is shown in Figs.8-9 respectively and corresponding slider displacement and cam profiles are shown in appendix Fig. A1toA4. From above discussion it is worth to say that CCM is more useful for industrial application.

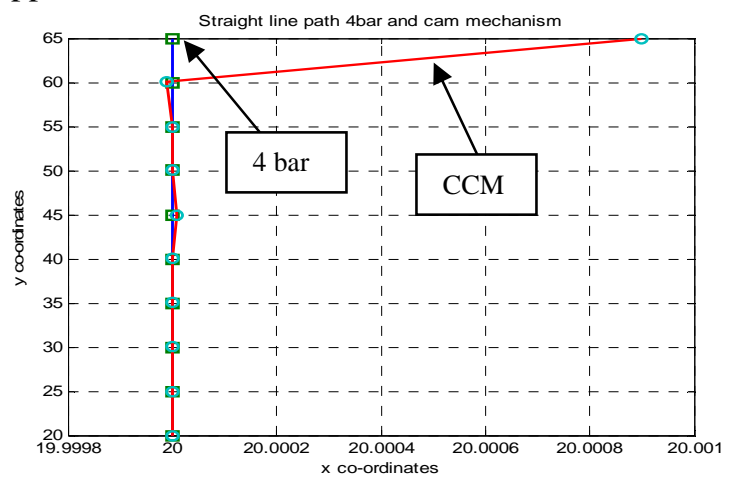

Figure 7. Straight line path

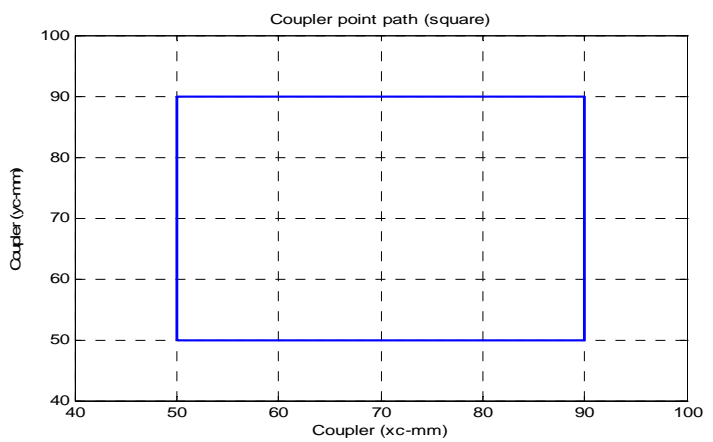

Figure 8. Square profile 


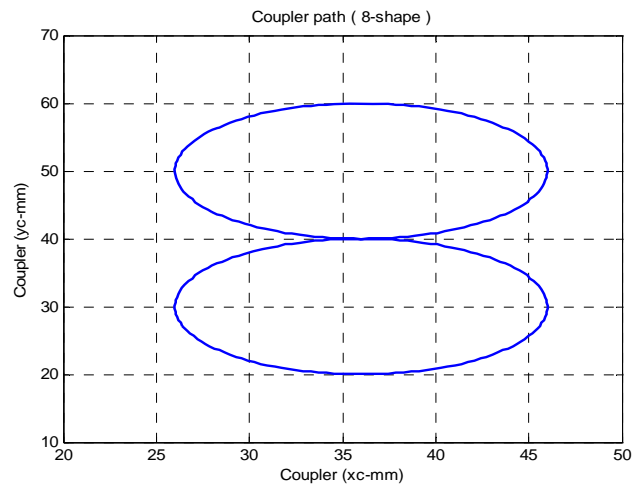

Figure 9. Double circle profile (8)

\section{Kinematic analysis of proposed CCM}

Kinematics deals with the study of relative motion between the various links of machine, ignoring the forces involve in producing that motion. Thus, kinematics is the study to determine the displacement, velocity and acceleration of the various links of a mechanism (Denavit, 1964, Sadler and Yang, 1994). This study is essential to analyze how the proposed mechanism transmitting or modifying the available mechanical energy to some kind of useful energy. Here, useful methods of the kinematic analysis of proposed CCM were discussed in following section. Very often, a mechanism with higher pairs can be replaced by an equivalent mechanism with lower pairs (Ghosh and Mallik, 2002, Shigley and Uicker, 1980). This equivalence is valid for studying only the instantaneous characteristics. The equivalent lower pair mechanism facilitates analysis as a certain amount of sliding takes place between connected in a higher pairs. Referring to Fig. 5, cam and follower contact forms higher pair. This pair was replaced in equivalent mechanism as per kinematic principle of motion transfer. Consequently kinematic equivalent of proposed CCM has been shown in Fig.10, for circular shape of coupler curve. Path of coupler point ' $T$ ' has finite and constant radius of curvature.
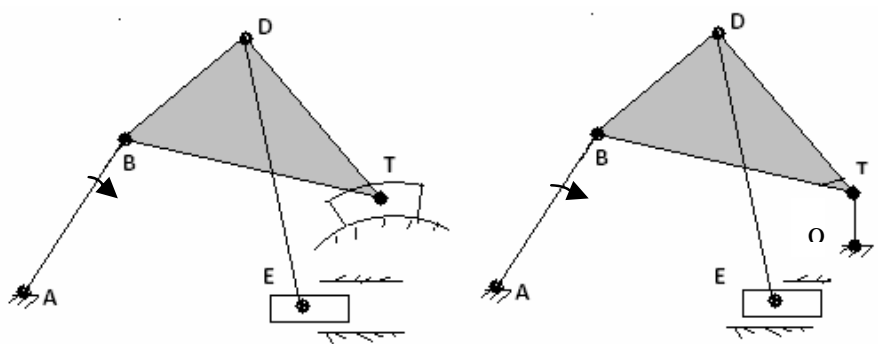

Figure 10. Kinematic equivalent mechanism for circular coupler path

Velocity of moving body is a vector quantity having the magnitude and direction. Rate of change of velocity is known as acceleration. Velocity and acceleration diagrams shown in Fig. 11, are drawn using a graphical method of relative point. Input link $\mathrm{AB}$ represents the cam, by knowing input velocity and acceleration following diagrams can be completed. Velocity and acceleration components are represented in Table. II

Table II. Components of velocity and acceleration (circular profile)

\begin{tabular}{|c|c|c|c|c|c|c|c|c|c|c|c|c|c|c|}
\hline $\begin{array}{l}\operatorname{lin} \\
k\end{array}$ & $v^{t}$ & fc & $\mathrm{ft}$ & fs & link & $\mathrm{v}^{\mathrm{t}}$ & fc & $\mathrm{ft}$ & fs & link & $\mathrm{v}^{\mathrm{t}}$ & fc & $\mathrm{ft}$ & fs \\
\hline$A B$ & $\begin{array}{l}B \\
A\end{array}$ & $\begin{array}{l}\mathrm{b} \\
\mathrm{a}\end{array}$ & - & - & $\mathrm{T}(\mathrm{OT})$ & TO & $\begin{array}{c}\text { xa } \\
\text { (xo) }\end{array}$ & tx & - & DE & ED & dy1 & yle & - \\
\hline BT & TB & by & ty & - & BDT & DT & xlt & $d x 1$ & - & $E$ & EO & - & - & $\begin{array}{c}\text { ea (eo } \\
\text { ) }\end{array}$ \\
\hline
\end{tabular}



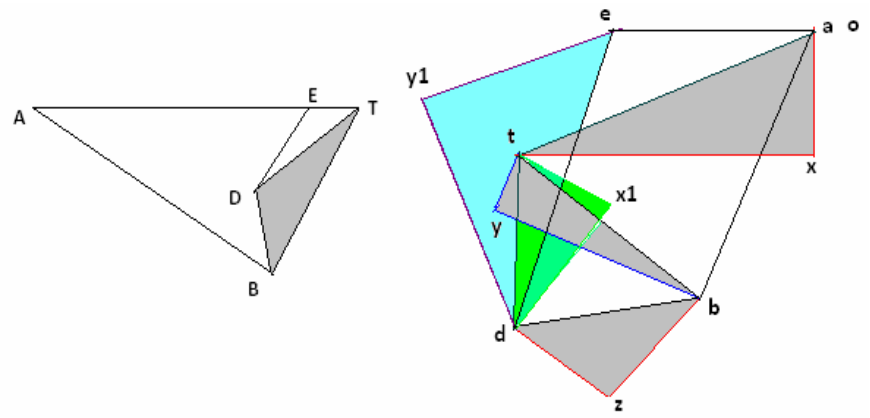

Figure 11. Velocity and acceleration diagrams for circular coupler path

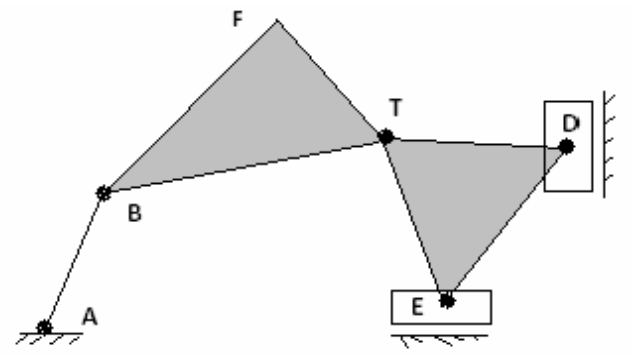

Figure 12. Kinematic equivalent mechanism for elliptical coupler path

Fig.12 shows kinematic equivalent of CCM for elliptical shape of coupler curve. If the radius of curvature of the paths traversed by motion transfer points of all links comprising a mechanism is known, the acceleration analysis of the mechanism can be completed by relative point method. But in this case, radius of curvature of the motion transfer point ' $T$ ' is varying therefore relative point method was not directly applicable. So resulting equivalent of CCM has low degree of complexity it was analyzed by using normal acceleration method. Figs. 13-14 show velocity and acceleration diagrams, drawn by normal acceleration method. The steps to be followed in applying this method are i) Transfer the mechanism in to a simple one by changing the input link as slider ' $E$ ' ii) Perform the velocity analysis with this alternative input link, and determine true velocities iii) Draw an auxiliary acceleration diagram based on true velocities and zero acceleration of the alternative input link. Determine the normal component of acceleration of the floating point ' $\mathrm{T}$ ' which has path of unknown radius of curvature iv) Construct the true acceleration diagram with the actual input acceleration, using the information obtained in above steps. Velocity and acceleration components are represented in Table. II

Table III. Components of velocity and acceleration (elliptical profile)

\begin{tabular}{|c|c|c|c|c|c|c|c|c|c|c|c|c|c|c|}
\hline $\begin{array}{l}\operatorname{lin} \\
k\end{array}$ & $v^{t}$ & fc & $\mathrm{ft}^{\mathrm{t}}$ & $\mathrm{fs}_{\mathrm{s}}$ & link & $\mathrm{v}^{\mathrm{t}}$ & fc & $\mathrm{ft}^{\mathrm{t}}$ & $f_{s}$ & link & $\mathrm{v}^{\mathrm{t}}$ & fc & $\mathrm{ft}$ & $\mathrm{fs}$ \\
\hline$E$ & $\begin{array}{l}E \\
A\end{array}$ & - & - & ea & $\mathrm{D}$ & $\begin{array}{l}D \\
A\end{array}$ & - & - & da & $\mathrm{T}$ & ta & xa & tx & - \\
\hline ED & $\begin{array}{l}\mathrm{D} \\
\mathrm{E}\end{array}$ & $\begin{array}{c}\text { ey } \\
1\end{array}$ & yld & - & $\begin{array}{c}\text { DT } \\
\mathrm{E}\end{array}$ & ET & tzl & zle & - & $A B$ & ba & ba & - & - \\
\hline & & & & & & & & & & BT & tb & $\mathrm{yb}$ & ty & - \\
\hline
\end{tabular}
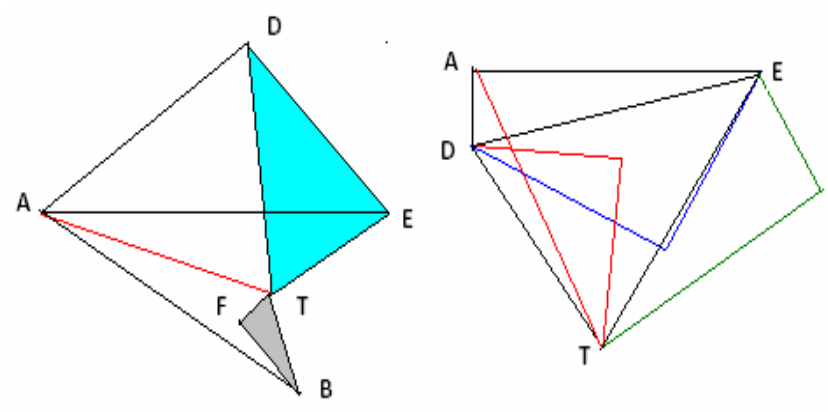

Figure 13.Velocity and auxiliary acceleration 

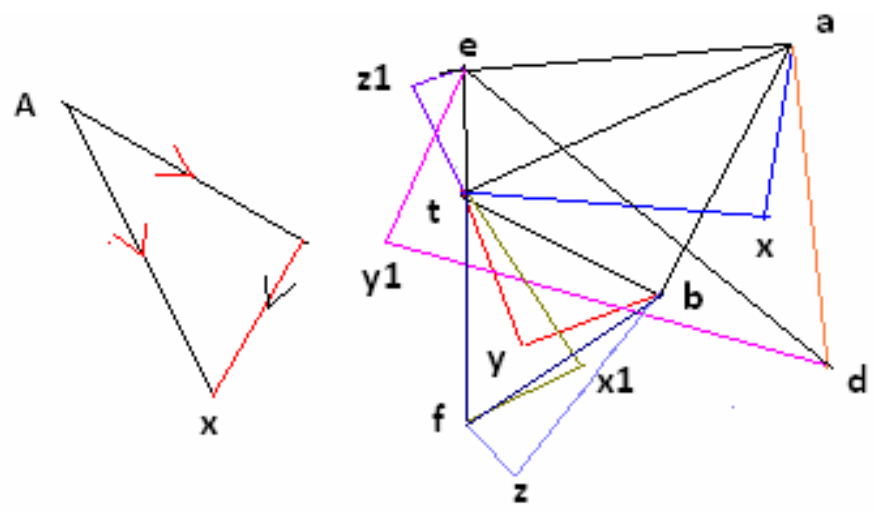

Figure 14.Actual acceleration

After completing these diagrams kinematic quantities (velocity and acceleration) of any link or any motion transfer points can be analyzed by doing vector calculation. This study shows how one can analyze proposed CCM for its equivalent mechanisms and kinematics.

\section{Dynamic stability of proposed CCM}

In proposed mechanism (Fig.5), interface joint between cam and follower (slider) is maintained during the backward portion of the cycle by the return spring. However, if during this period negative acceleration is too high the joint separates, resulting in the undesirable phenomenon known as follower jump. A common way to analyze a follower jump is to create the single-degree of freedom model shown in Fig. 15. In this type of model, $F_{i}(t)$ is the cam follower interface force, $k_{e q}$ represents an equivalent linear spring stiffness of the system, $C_{e q}$ linear viscous damper and $m_{e q}$ an equivalent system of mass. Detail process of model creation can be found in (Norton, 2002, Chen, 1980). Applying Newton's second law of motion to the system results

$$
F_{i}(t)=m_{e q} \ddot{x}+c_{e q} \dot{x}+k_{e q}\left(x+x_{0}\right)
$$

Where $x_{0}$ is the initial spring deflection that creates the spring preload force $F_{0}=k_{e q} x_{0}$ The superimposed dots in equation (6) represent time derivatives. Also, in the simplified model, we assume that $x(t)=s(t)$. Therefore, relationships between kinematic and true quantities, equation (6) can be written as

$$
F_{i}(t)=\omega^{2} m_{e q} s^{\prime \prime}(\theta)+\omega c_{e q} s^{\prime}(\theta)+k_{e q}\left(s(\theta)+s_{0}\right)
$$

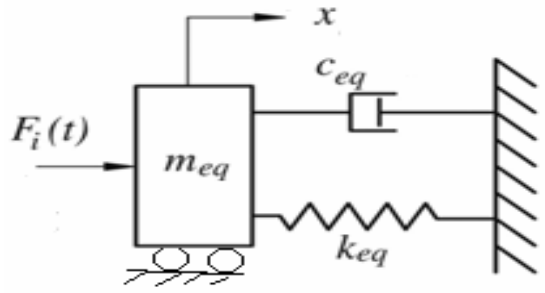

Figure.15 Single degree freedom model

To maintain the interface joint requires that $F_{i}(t)$ be greater than zero. Impending follower jump is predicted when the interface force goes to zero. Furthermore, because of the $\omega^{2}$ factor, the acceleration is especially important in reducing the interface force during the fall portion of the cycle. 


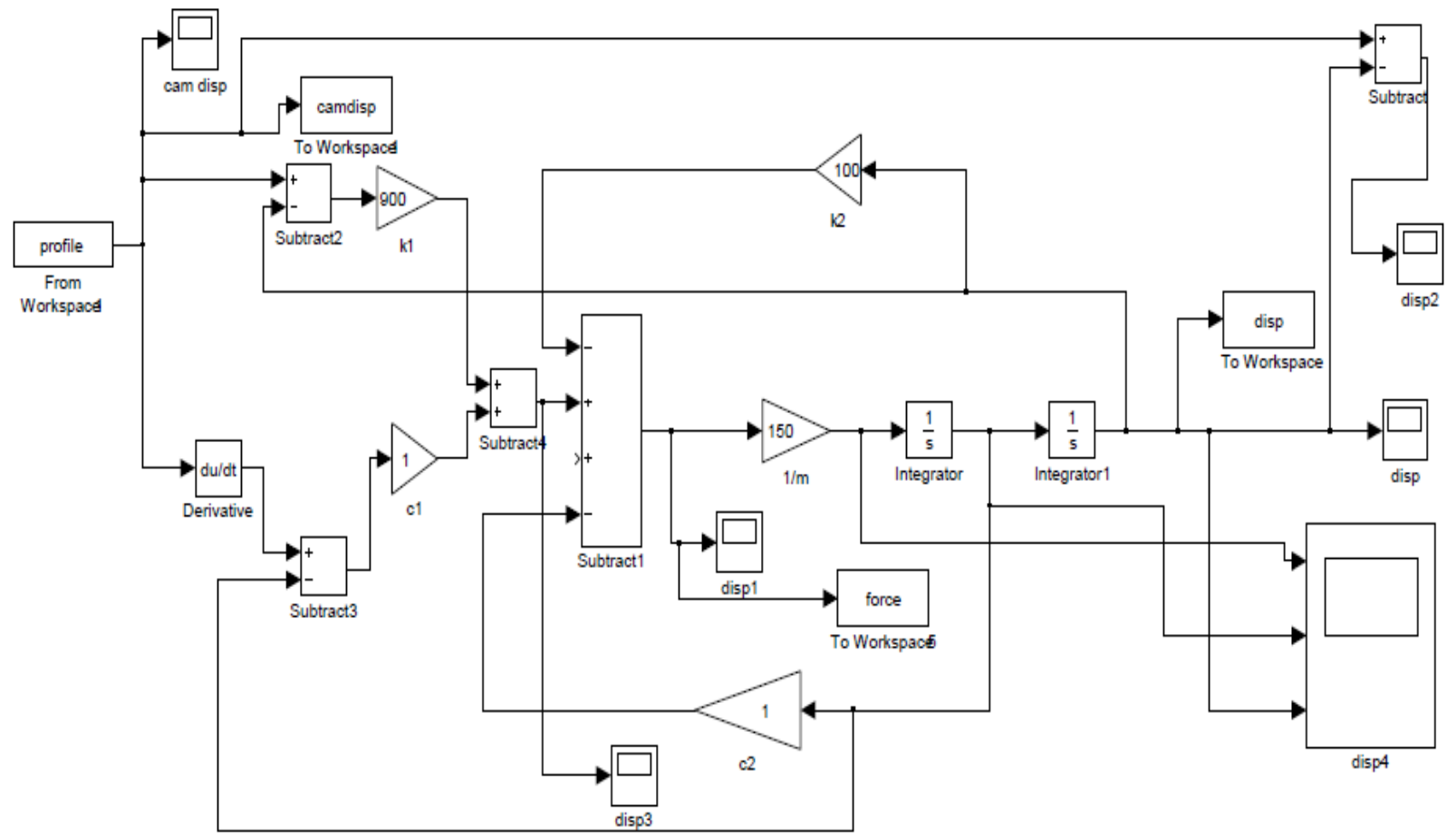

Figure 16.Matlab model

MATLAB is the suitable tool for computation and mechanism simulation. In this work, simulation using MATLAB progamming and SIMULINK toolbox was perform to study the dynamic stability of proposed CCM. Matlab script files and simulink model were developed shown in Fig. 16.

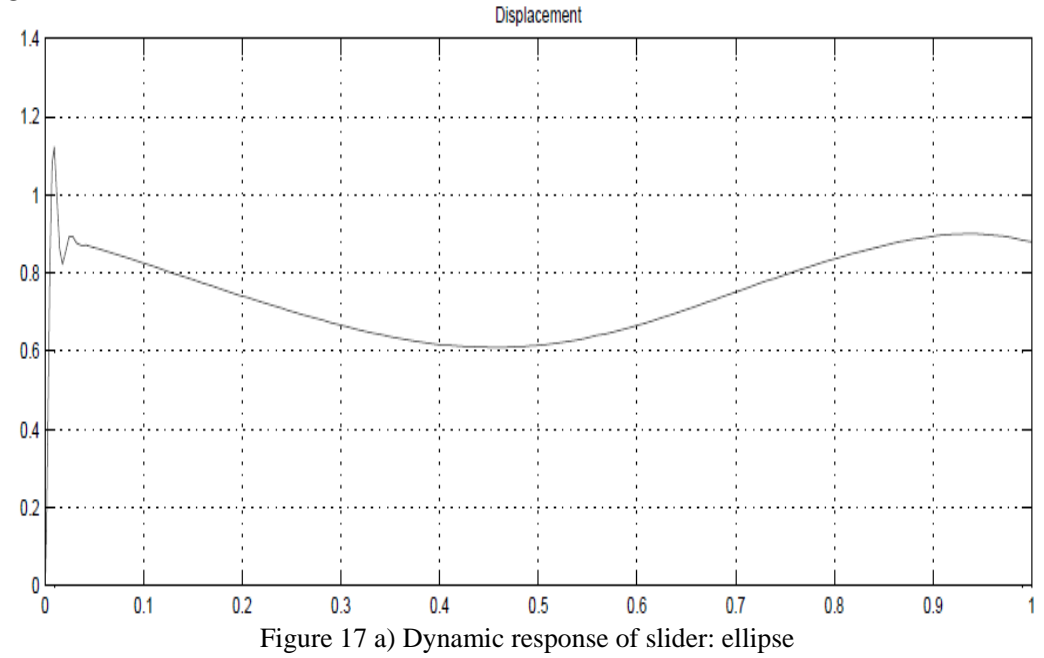

Fig.17 a-c shows dynamic response of slider in various cases of coupler curves. It is observed that trends of dynamic response and kinematic curves (shown in Fig.3, A1 and A3) similar. It means that well concurrence between kinematic and dynamic quantities of all cases. Also there is no separation of interface joint between cam and slider 

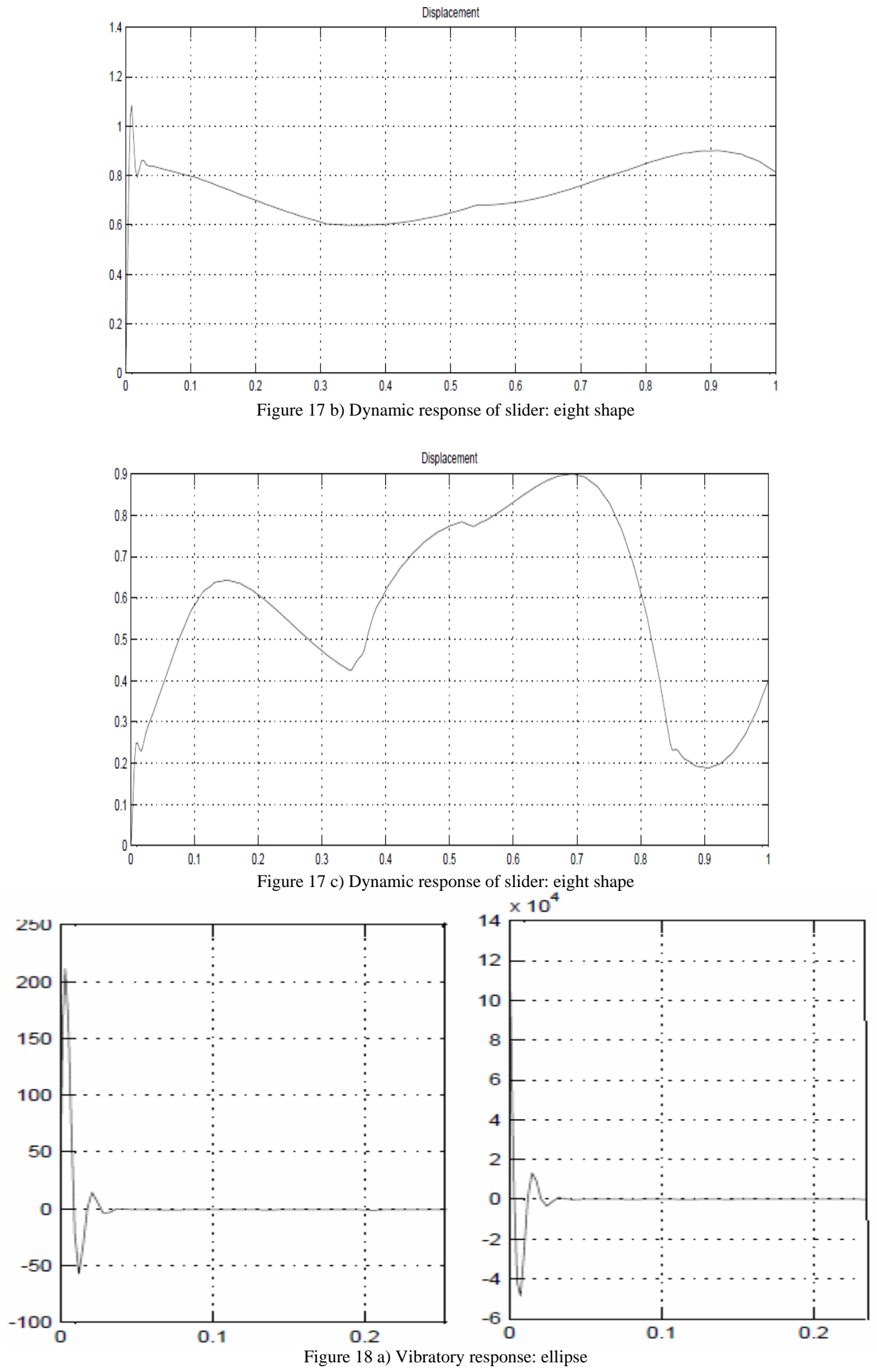

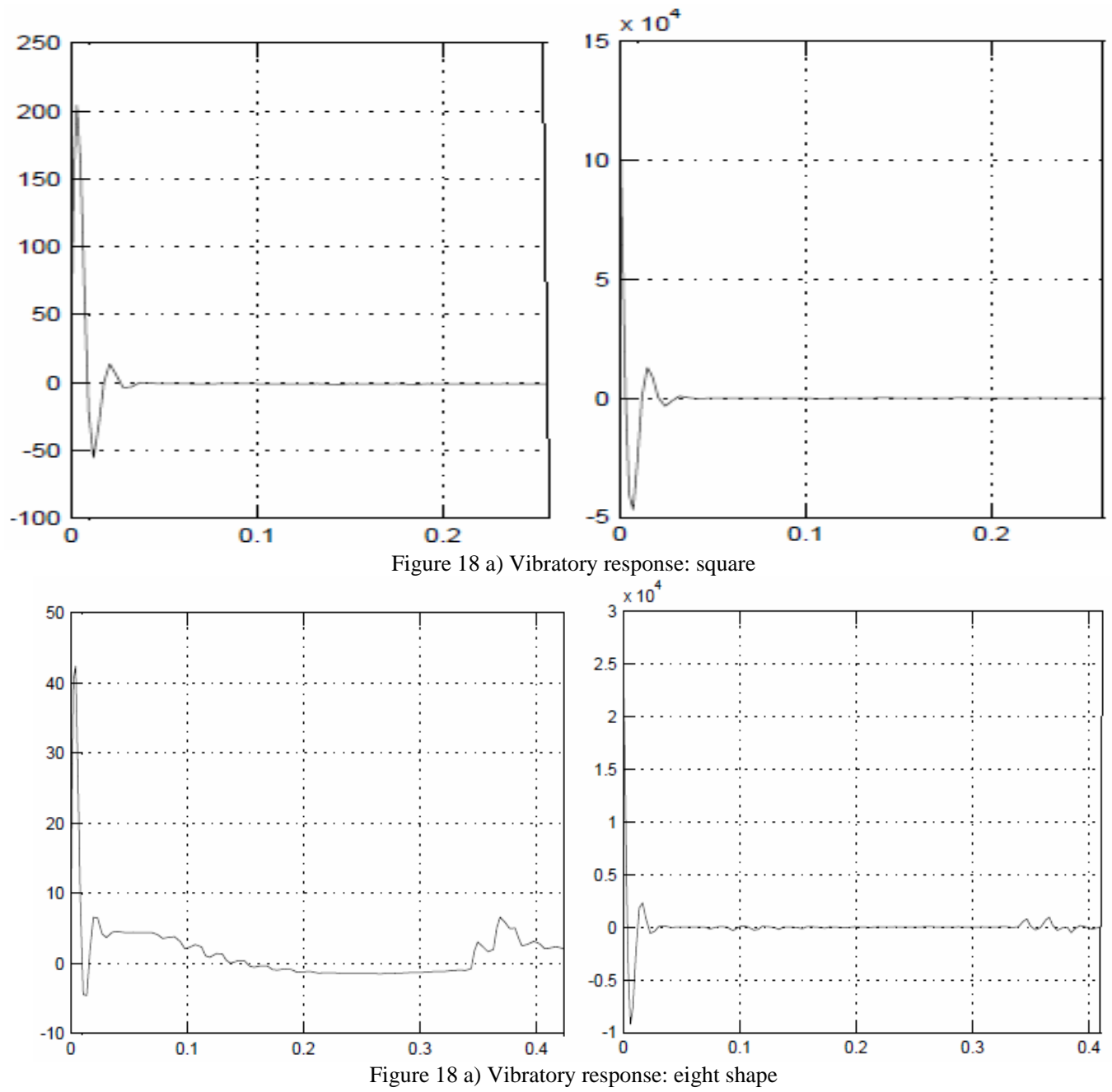

Fig.18 a-c shows vibratory response of a proposed CCM in various cases of coupler curves. In each case time-amplitude plots and their derivative were studied. It is observed that motion of mechanism reach to stable state (less or zero amplitude) quickly. This property is highly desirable for any mechanical system. Thus, this analysis shows that proposed mechanism is dynamically stable.

\section{Conclusion}

A new mechanism has been proposed for precise and complex path generation with infinite number of precision points. Resulting mechanism was kinematical compound and low degree of complexity. Kinematic and dynamic point of view mechanism found stable. In industrial applications to generate the coupler curves in different shapes, proposed combined planar cam mechanisms are suitable. Genetic algorithm technique can be used effectively in the synthesis of mechanisms. Future work will be to develop CCM for automotive valve train applications, including variable lift and timing.

\section{Nomenclature}

$x_{t}, y_{t}$ - Precision or target points (coordinates of cutting shape or profile) in $x$ and $y$ direction

$S, S_{\max }, S_{\min }$ - Slider displacement

$r \quad$ - Link length

$x, \dot{x}, \ddot{x} \quad$ - Dynamic displacement, velocity and acceleration

$\omega$ - Angular velocity of cam

$v^{t}$ - Tangential velocity

$f^{c}$ - Centripetal acceleration

$f^{t}$ - Tangential acceleration

$f^{s}$ - Sliding acceleration 


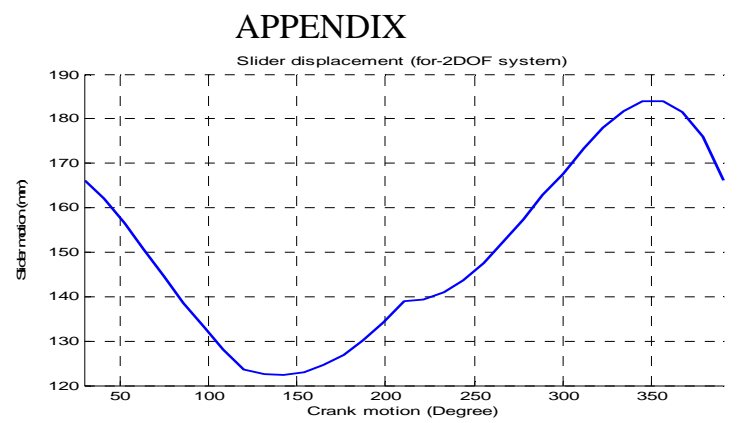

Figure A1. Slider displacement (square)

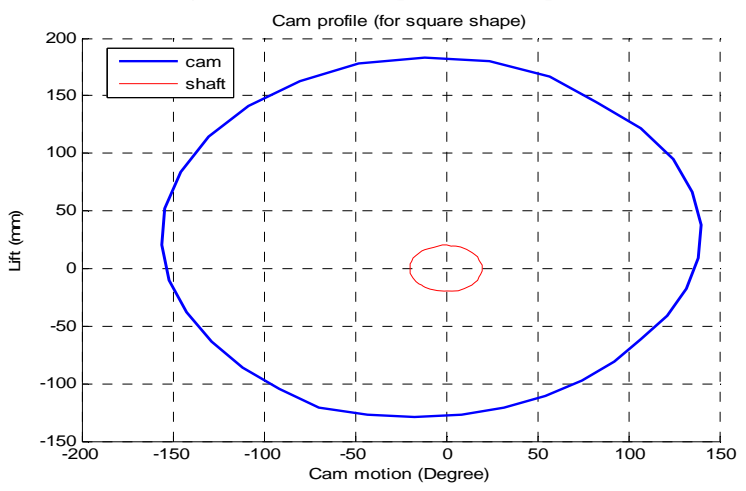

Figure A2. Cam profile (square))

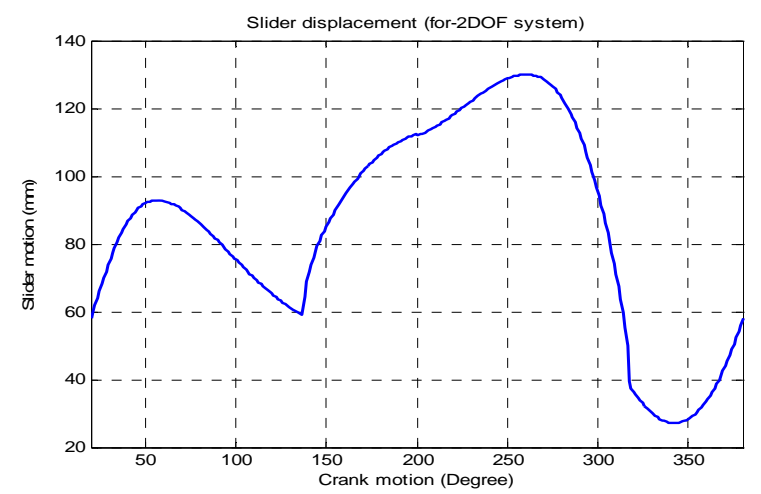

Figure A3. Slider displacement (8-profile)

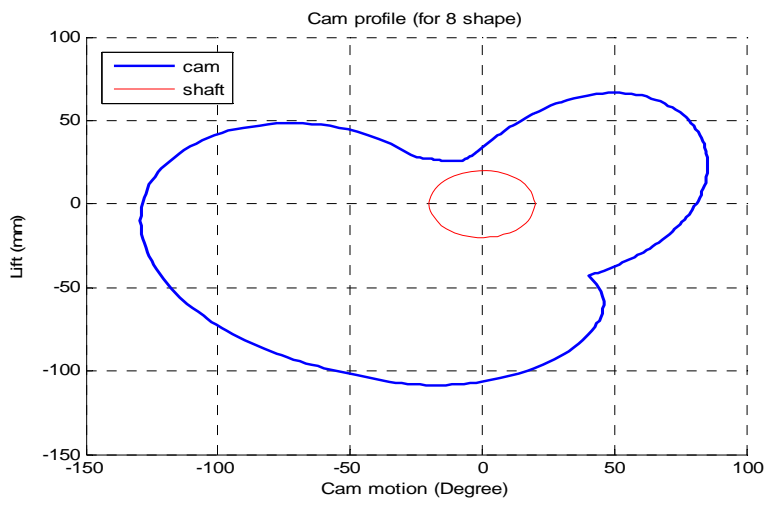

Figure A4. Cam profile (8-profile)

\section{Acknowledgment}

The author would like to acknowledge the University of Pune, BCUD for financial support. (Grant No: BCUD/OSD/184/09) 


\section{References}

Angeles, J., and Carlos Lopez C.,1988. Optimal synthesis of cam mechanisms with oscillating flat-face followers. Journal of Mechnism and Machine Theory, Vol. 23, No. 1, pp. 1-6.

Chen, F. Y. 1982. Mechanics and Design of Cam Mechanisms, Pergamon, New York

Erdman, A.G. 1981.Computer aided mechanism design. Journal of Mechanical Design, Vol.117, pp. 93-100.

Erdman, A.G. 1991.Three and four precision point kinematic synthesis of planar linkages. Journal of Mechnism and Machine Theory, Vol. 16, No. 5, pp. 227-245.

Freudenstein, F. 1954. An analytical approach to the design of four-link mechanisms. Trans. of the ASME, Vol. 76, pp. 483-492.

Fang, W.E. 1994. Simultaneous type and dimensional synthesis of mechanisms by GA. Mechanism Synthesis and Analysis, Vol. 70.

Gabrera, J.A., Simon, A., and Prado, M. 2000. Optimal synthesis of mechanisms with genetic algorithms. J Mech and Mach

Theory, Vol. 37, pp.1165- 1177.

Gen, M., and Cheng, R. 2000. Genetic algorithms and engineering optimization. John Wiley, Singapore.

Ghosh, A., and Mallik, A. K. 2002. Theory of machines and machines, affiliated east west press, New Delhi.

Goldberg, D.E. 1989. Genetic algorithms in search, optimization and machine learning. Addison Wesley, Massachusetts.

Hartenberg, R., and Denavit, J. 1964. Kinematic synthesis of linkages. McGraw-Hill, New York.

Hafez, Tari., and Hai-Jun, Su. 2010. Complete Solution to the Eight-Point Path Generation of Slider-Crank Four-Bar Linkages.

Journal of Mechanical Design, No 8, pp 94-100.

Kunjur, A., and Krishnamurty, S. 1997. Genetic algorithms in mechanical synthesis. Journal Applied Mech and Robotics, Vol. 4

2, pp. 18-24.

Lampien, J. 2003. Cam shape optimization by genetic algorithm. Journal of Computer Aided Design, Vol. 35pp.727-737.

Laribi, M. A., Mlika, A., Romdhane, L., and Zeghloul, S.A. 2004. Combined genetic algorithm-fuzzy logic method (GA-FL) in Mechanism synthesis. Journal of Mechnism and Machine Theory, Vol. 39, pp. 717-735.

Mundo, D., Liu, J.Y., and Yan, H.S. 2006. Optimal synthesis of cam-linkage mechanisms for precise path generation. Journal of Mechanical Design, Vol. 128, pp. 1253-1260.

Norton, R. L. 2002. Cam Design and Manufacturing Handbook, Industrial Press, New York.

Sadler, J.P., and Yang, Z. 1990. Optimal design of cam-linkage mechanisms for dynamic-force characteristics. Journal of Mechnism and Machine Theory, Vol.25, pp. 41-57.

Saggere, L., and Kota. 2001. Synthesis of planar, compliant four-bar mechanisms for compliant-segment motion generation. Journal of Mechanical Design, Vol. 123, pp. 535-541.

Sandor, G.N., and Erdman, A.G. 1984. Advanced mechanism design, analysis and synthesis. Prentice-Hall, New Jersey.

Sandor, G.N., and Erdman, A.G. 1991. Mechanism design, analysis and synthesis. Prentice Hall, Englewood Cliffs.

Singh, Y.P., and Kohli, D. 1981. Synthesis of cam-link mechanisms for exact path generation. Journal of Mechnism and Machine Theory, Vol.16, pp. 447-457.

Shigley, J. E.,and Uicker Jr. J.J. 1980. Theory of Machines and Mechanisms, McGraw-hill, New York.

Saxena, A. 2005. Synthesis of compliant mechanisms for path generation using genetic algorithm. Journal of Mechanical Design, Vol. 127, pp. 745-752.

Ullah, I., and Kota, S. 1997. Optimal synthesis of mechanisms for path generation using Fourier descriptors and global search methods. Journal of Mechanical Design, Vol. 119, pp. 504-510.

Ye, Z., and Smith, M. R. 2005. Design of a combined cam-linkage mechanism with an oscillating roller follower by an analytical method. Journal of Mech Engg Science, Vol. 219, pp. 419-427.

Umesh, Chavan., Mukund, Nalawade., and Satishchandra Joshi. 2010. Synthesis of coupler curves with combined planar cam follower mechanisms by genetic algorithm. Proceeding of $2^{\text {nd }}$ International Conference on Computer Engineering and Technology, Chengdu, China

\section{Biographical notes}

Prof. U.S. Chavan, Associate Professor at Vishwakarma Institute of Technology, Pune. Received M.E. in Mechanical Engineering with Machine Design as specialization, from College of Engineering Pune (COEP) Pursuing Ph.D. from University of Pune, India Research interest includes dynamics of mechanical systems, synthesis, design and simulation. 14 years of teaching and research experience in various subjects of Mechanical Engineering, guided about 12 M.E. thesis and number of B.E. projects. Around 8 publications in various National and International Journals and Conference Proceedings

Prof. Dr. S.V. Joshi, Ph.D., Professor in Mechanical Engineering at Vishwakarma Institute of Technology, Pune. He received his M.E. in Mechanical Engineering, from Asian Institute of Technology, Thailand and Ph.D from Indian Institute of Technology, Bombay. He has an experience of 15 years in teaching and research in various subjects of Mechanical Engineering. He also has 17 years industrial experience. He has guided number of M.E. thesis and B.E. projects. Currently, six Ph.D students are working under his supervision. He has around 21publications to his credit in various National and International Journals and Conference Proceedings. 